\title{
Questions on nanoplastic
}

\author{
Research on nanoplastic has already provided some significant results but it has also exposed a large number of
} open questions.

A simple look at the rapidly increasing number of publications and grants started every year since 2016 (see figure) indicates that nanoplastic has become a proper research field. This is only natural, considering the growing concern caused by the observation of plastic fragments of all sizes and shapes in virtually every kind of environment. Indeed, the term microplastic has been used for quite some time to refer to specimens smaller than $5 \mathrm{~mm}$ that result from the degradation of large pieces of plastic dispersed in the environment. The possibility that such specimens degrade into submicrometre particles has attracted the attention of both environmental scientists and toxicologists working on plastic pollution as well as those working on engineered nanomaterials.

The Perspectives by Denise Mitrano and co-workers and Julien Gigault and co-workers, now published in Nature Nanotechnology aim at placing the potential risks posed by nanoplastic to humans and the environment within the wider context of emerging contaminants. They both provide a useful structure to organize the knowledge that the research community has gained so far, and perhaps more importantly, the types of questions that still need to be answered. We refer you to the two Perspectives for all the details but we'd like to summarize here the points that both Perspectives touch upon and that in our view should be kept in mind when discussing nanoplastic.

The first issue to consider is whether it is at all necessary to distinguish nanoplastic from microplastic; namely if the distinction reflects different physical and chemical properties or it is simply a categorization born of convenience. Based on what we know so far, and as illustrated in both Perspectives, it is fair to say that indeed smaller particles interact differently with light, diffuse differently in the environment and penetrate more effectively into organisms. In other words, their environmental fate and the potential

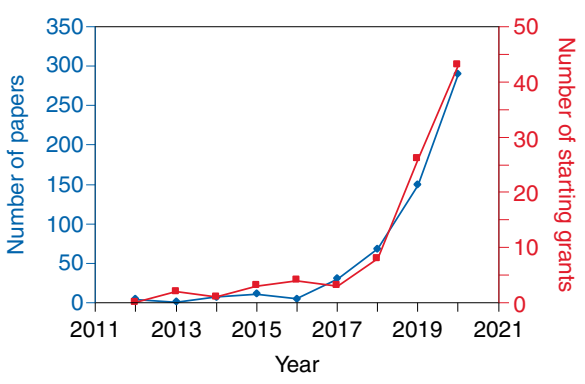

Number of publications with the word 'nanoplastic' or 'nanoplastics' in the title or abstract, and number of starting grants on nanoplastic. Data from SNInsights, extracted 27 April 2021.

risks of nanoplastic are bound to be different from those of microplastic. As it is the case for engineered nanomaterials, there is no universal size at which the properties change. Rather, this will depend on the type of plastic considered. Furthermore, the changes are not necessarily abrupt. But at least scientifically it makes perfect sense to consider nanoplastic and microplastic as distinct.

A second point to consider is whether the risk posed by nanoplastic is likely to be as high as perhaps feared by public opinion. The most appropriate answer is that it is simply too soon to tell. A number of studies have shown that nanoplastic can penetrate both animal and plants, and there is some evidence of negative effects. But aside from the small number of such results, the potential heterogeneity in both size and composition of nanoplastic fragments makes it impossible to generalize at this stage.

Connected to the previous point is the estimate of the amount of nanoplastic present in the environment because eventually this will influence heavily the risk posed to the organism. Nanoplastic particles are incidental in nature and their number cannot be estimated based on the amount of manufactured material, like it can be attempted for engineered nanomaterials, for example. At least for now it is simply too difficult to establish how much plastic waste eventually degrades into nanoplastic. One effective way forward is to establish analytical techniques that can provide reliable accounts of nanoplastic concentrations in different environments, but despite considerable efforts these are still lacking.

In the absence of reliable techniques that can be used to study the environmental fate of nanoplastic, several groups have developed the synthesis of model nanoplastic particles that can be easily tracked and therefore used to monitor how they diffuse and transform, especially in water, soil and when in contact with different organisms. We believe that these types of studies are very valuable and will continue to provide essential information. The question remains however on how representative these model systems are, considering the wide heterogeneity in shapes, size and composition that environmental nanoplastic exhibits and that has been reproduced artificially only partially so far. Perhaps the development of techniques aimed at reproducing such diversity in model systems may be an effective way forward.

We have established that it makes sense to consider nanoplastic as a different entity from microplastic. As the two Perspectives highlight, nanoplastic particles are also quite different from engineered nanomaterials. Still, the expertise and interest of researchers in environmental nanotechnology and in plastic pollution will prove invaluable to provide a better picture. In the meantime, we invite you to attend our webinar on 19 May, during which we discuss the above issues and others with experts in the field (https://www.nature.com/nnano/video). See you there.

Published online: 13 May 2021 https://doi.org/10.1038/s41565-021-00918-z 